\title{
DISEÑO DE REPOBLACIONES FORESTALES EN ZONAS ÁRIDAS: TAMAÑO DEL MICROEMBALSE Y RELACIÓN ENTRE EL ÁREA DE IMPLUVIO Y EL ÁREA DE RECEPCIÓN
}

\author{
J. Mongil Manso ${ }^{1}$,A. Martínez de Azagra² \\ 1 Área Agroforestal y Ambiental \\ Universidad Católica de Ávila \\ 2 Unidad de Hidráulica e Hidrología. E.T.S. Ingenierías Agrarias \\ Universidad de Valladolid
}

\section{RESUMEN}

En el momento de proyectar cualquier reforestación en una zona árida situada en ladera, la economía del agua debe jugar un papel preponderante. La consecución de cosechas de agua para los brinzales en sus primeras etapas de vida resulta crucial para el éxito de la empresa. En este trabajo se desarrollan algunos criterios —basados en el modelo hidrológico MODIPÉ- para fijar el tamaño de la unidad sistematizada y de las trampas de agua a crear con la preparación del suelo. Estos criterios sirven para orientar al técnico encargado de la restauración sobre la densidad de plantación a introducir y sobre el tamaño de los alcorques a realizar, todo ello con vistas a aumentar la supervivencia del repoblado reduciendo la alteración del microrrelieve a lo mínimo indispensable.

Palabras clave: Cosechas de agua, repoblación forestal, zonas áridas, oasificación.

\section{SUMMARY}

Water economy plays a most important role when reforesting slopes in arid zones. Convenient water harvesting techniques allow the seedlings to survive, establish and grow much better due to a beneficial microclimate. In this paper we develop some basic criteria to fix the size of the water traps and microcatchments to be created with an adequate soil preparation. These criteria should help forest technicians to choose planting density and micropond size combining both: best survival chances of the seedlings and minimal alterations of the original microtopography of the slope.

Key words: Water harvesting, reforestation, arid zones, oasification. 


\section{Introducción}

Un sistema de recolección de agua consiste en dos partes: un área donde se induce y genera la escorrentía superficial (área de impluvio o productora) y un área en donde se recogen y almacenan dichos aportes hídricos (área de recepción o colectora). Es en esta área donde se instala un cultivo agrícola o se realiza una siembra o plantación de especies forestales. Las plantas se desarrollan mejor gracias al microclima favorable inducido.

La mayor parte de los métodos de preparación del suelo en el sector forestal persiguen obtener cosechas de agua con las que invertir procesos de desertificación por aridez edáfica (Martínez de Azagra, 1996). Al aumentar la infiltración en la ladera se favorece la instalación de una vegetación más tupida, especialmente en climas áridos. Este proceso ha sido denominado por los autores como oasificación (Martínez de Azagra, 2000; Martínez de Azagra et al. 2004), que consiste en la construcción de pequeñas estructuras de tierra para recoger e infiltrar la precipitación y la escorrentía, por modificación de la fisiografía de las laderas de forma adecuada y no impactante (véase www.oasificacion.com). De esta manera se pueden mejorar las condiciones hídricas del suelo y el establecimiento y desarrollo de una vegetación leñosa que invierta el peligroso proceso de desertificación (véase la figura 1).

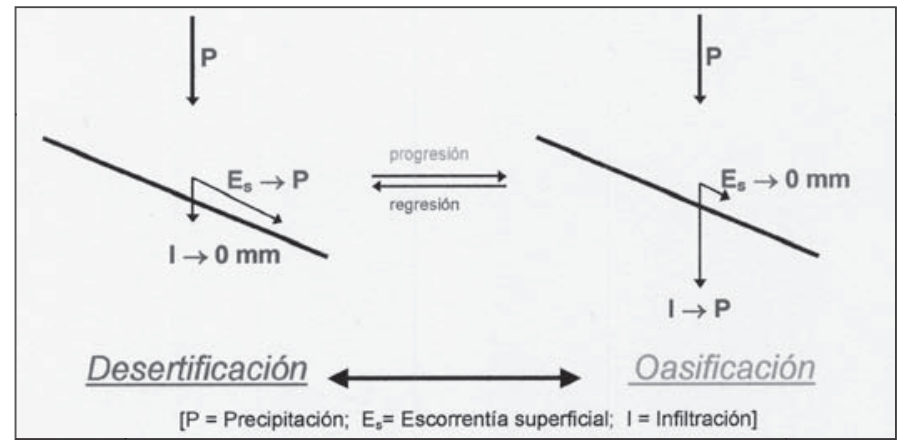

Figura 1. Progresión hídrica, edáfica y vegetal desde una ladera degradada hasta una restaurada.

El modelo hidrológico MODIPÉ (Martínez de Azagra, 1996) puede resultar muy útil para el correcto diagnóstico de muchos procesos de desertificación y para una acertada toma de decisiones a la hora de restaurar laderas degradadas. Entre otros fines, puede utilizarse para evaluar el efecto hídrico de distintos procedimientos de preparación del suelo en repoblaciones forestales o para diseñar trampas de agua con las que se incrementa la infiltración y arraigo de repoblaciones.

En el presente artículo, basándonos en el modelo anteriormente mencionado y teniendo muy presente la economía del agua, ofrecemos unos criterios de referencia con los que diseñar una repoblación forestal en laderas de zonas áridas y semiáridas. Estos criterios deben servir al técnico encargado de proyectar la repoblación para determinar la densidad de plantación y el tamaño del microembalse a construir, alterando lo mínimo posible el microrrelieve de la ladera. 


\section{Tamaño del microembalse}

Cuando se pretenda proyectar una repoblación forestal en una zona árida situada en ladera, la economía del agua debe jugar un papel preponderante. La obtención de cosechas de agua para los brinzales en sus primeras etapas de vida resulta fundamental para el éxito de la empresa.

La mayor parte de los métodos de preparación del suelo utilizados en el sector forestal persiguen obtener cosechas de agua con las que invertir procesos de desertificación por aridez edáfica. Al aumentar la infiltración en la ladera se favorece la instalación de una vegetación más tupida, especialmente en climas áridos.

Pero para lograr el objetivo restaurador deseado con una repoblación, conviene dimensionar correctamente el microembalse, es decir el recipiente creado mediante un movimiento de tierras que dotará de agua a la planta. El tamaño del microembalse puede fijarse atendiendo a diferentes criterios. Muchos de ellos están íntimamente relacionados entre sí, otros son complementarios y hasta opuestos. Una adecuada conjugación de estos criterios conduce a la solución más oportuna en cada caso. Estos criterios deben servir para orientar al técnico encargado de la restauración sobre el tamaño de los alcorques a realizar, todo ello con la intención de aumentar la supervivencia del repoblado reduciendo la alteración del microrrelieve a lo mínimo indispensable.

La mayoría de los trabajos consultados sobre cosechas de agua no prestan especial atención al tamaño que debe tener el microembalse que se sitúa en el área colectora. Los diferentes autores que se han ocupado de este tipo de técnicas han hecho especial hincapié en la superficie de este área y su relación con el área de impluvio, pero en pocos casos tratan de la altura que deben tener los muretes o caballones que conforman la estructura recolectora de escorrentía. En esta línea se sitúa el excelente trabajo recopilatorio de Boers \& Ben-Asher (1982), por ejemplo. Critchley \& Siegert (1991) van algo más lejos, haciendo recomendaciones de las dimensiones que deben tener los caballones en las diferentes estructuras tradicionales de recolección de agua que describen. Así, por ejemplo, para las microcuencas Negarim, recomiendan, desde la experiencia, alturas de caballón desde 25 hasta $55 \mathrm{~cm}$, dependiendo del tamaño de la microcuenca y de la pendiente del terreno.

Por otra parte, el modelo MODIPÉ (Martínez de Azagra, 1995) cuantifica el tamaño del microembalse a través de la variable denominada $M A X$, que es la capacidad mínima de embalse para recoger toda la escorrentía superficial que se produce en la unidad sistematizada, es decir, para conseguir endorreísmo. Las ecuaciones que se utilizan para calcular esta capacidad de alcorque pueden consultarse en el apartado 2.5 del presente trabajo. No obstante, el modelo MODIPÉ atiende exclusivamente a razones de economía del agua a la hora de aconsejar el tamaño del embalse, resultando interesante tener en cuenta otros aspectos. Y, por otro lado, no desarrolla una metodología completa sobre el particular. Por este motivo, Martínez de Azagra \& Mongil (2001), basándose en este modelo, han esbozado unos criterios a seguir para el adecuado dimensionado de un microembalse, que son los que ahora se desarrollan en profundidad.

El método que se propone para determinar el tamaño óptimo del microembalse se basa en doce criterios diferentes. Se trata de una serie de condicionantes o factores que deben considerarse en el diseño del alcorque. Algunos de ellos están íntimamente relacionados entre sí, otros son complementarios y hasta antagónicos. Por ello, sólo una correcta conjugación de todos los criterios lleva a la solución más adecuada en cada caso concreto. Los criterios son los siguientes: 


\section{1) Criterio hidrometeorológico}

Para que el microembalse cumpla su función, debe producirse endorreísmo para una determinada precipitación, serie de precipitaciones o conjunto de precipitaciones anuales; es decir, todo el agua que llega al área de recepción debe quedarse e infiltrarse en ella. Para conseguir esta situación, el procedimiento de cálculo del microembalse es el siguiente:

$1^{\circ}$ ) Ajuste de la serie de precipitaciones máximas diarias anuales a la distribución de Gumbel, recomendada para valores extremos, y realización del correspondiente test de bondad de ajuste de Kolmogorov-Smirnov.

$2^{\circ}$ ) Mediante el modelo MODIPÉ, obtención de una tabla en la que se relacionen las precipitaciones límite de la unidad sistematizada (la precipitación límite es el aguacero que genera un volumen de escorrentía máximo capaz de ser retenido en su totalidad por el área colectora), para diferentes capacidades de microembalse (CAPA), con sus correspondientes periodos de retorno calculados con la distribución de Gumbel.

$3^{\circ}$ ) Cálculo de CAPA (y altura de los muretes) para lograr endorreísmo con un periodo de retorno de 10 años (que se considera, aproximadamente, la vida útil de la preparación del suelo).

\section{2) Criterio de proporcionalidad con el área de impluvio}

El área de impluvio y el tamaño del microembalse deben estar relacionados, ya que el volumen de agua producido en la primera debe quedar retenido en un microembalse de volumen adecuado. De esta manera se asegura también el endorreísmo en la unidad sistematizada.

En el modelo MODIPÉ, el tamaño necesario o de referencia para el microembalse se determina mediante la variable denominada $M A X$, que es el volumen de agua que escurriría de la unidad sistematizada si la capacidad del microembalse fuese nula (con CAPA =0). MODIPÉ calcula MAX de dos maneras diferentes, dependiendo de si el número de curva del área de impluvio es mayor o menor que el del área de recepción (Martínez de Azagra, 1995). Empleando estas ecuaciones y considerando que $R$ es la relación entre el área de impluvio y el área de recepción $\left(R=S_{1} / S_{2}\right)$, se llega a las ecuaciones que sirven para calcular el tamaño del microembalse:

a) Para NI $\geq N R$

$$
\frac{M A X}{S_{2}}=\frac{(P-P 0)^{2}}{P+4 \cdot P 0} \cdot R+\frac{(P-P 0)^{2}}{P+4 \cdot P 0}=\frac{(P-P 0)^{2}}{P+4 \cdot P 0}(R+1)
$$

b) Para NI $<$ NR

$$
\frac{M A X}{S_{2}}=\frac{(P-P 1)^{2}}{P+4 \cdot P 1} \cdot R+\frac{(P-P R)^{2}}{P+4 \cdot P R}
$$

en donde:

$M A X=$ Escorrentía máxima que puede escapar de la sistematización para alcorque nulo (l)

$S_{1}=\quad$ Superficie del área de impluvio $\left(\mathrm{m}^{2}\right)$

$S_{2}=\quad$ Superficie del área de recepción $\left(\mathrm{m}^{2}\right)$ 


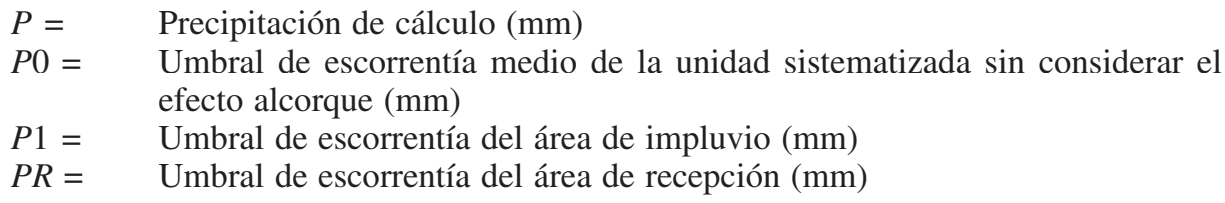

Con estas ecuaciones y conociendo la relación entre el área de impluvio y el área de recepción, o los valores de estos, la precipitación de cálculo y los umbrales de escorrentía, se calcula el tamaño necesario del microembalse proporcional al área de impluvio. La capacidad resultante del microembalse será exactamente la necesaria para recoger toda la escorrentía producida y no infiltrada para unas determinadas precipitaciones de cálculo.

\section{3) Criterio de resguardo hidráulico}

Según lo establecido por el criterio hidrometeorológico, se trata ahora de construir un microembalse algo mayor de lo estrictamente necesario. Es decir, una micropresa con un resguardo ante un determinado aguacero de cálculo o un cierto volumen de seguridad frente al desbordamiento, que suponga una disminución del número de curva equivalente $(N E Q)$ en varios puntos. Si el agua vertiera por encima del dique de tierra del caballón, debido a que está construido con materiales sueltos, los daños serían cuantiosos, reduciéndose considerablemente su vida útil.

Un paralelismo con las grandes presas tradicionales de tierra se muestra en la figura 2. En el caso del murete de un microembalse para repoblación forestal, los objetivos del resguardo son:

- Permanecer del lado de la seguridad en la consecución de endorreísmo en la microcuenca.

- Corregir el efecto de la distorsión que puede producirse debido a la escala manejada, ya que el método del número de curva, empleado por el modelo MODIPÉ, está calibrado para cuencas de varios kilómetros cuadrados.

- Considerar la reducción de la capacidad del microembalse que se produce debido al aterramiento parcial del microembalse y al achatamiento de los muretes con las primeras lluvias (aunque esta segunda circunstancia se tiene en cuenta en el criterio mecánico de asentamiento, que se expondrá posteriormente).

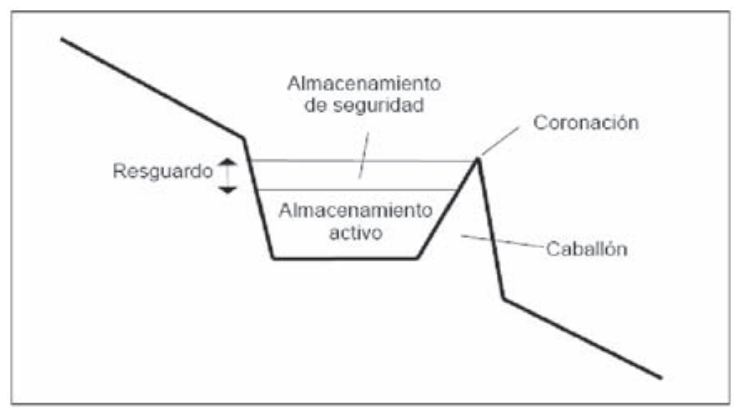

Figura 2. Zonas de almacenamiento y delimitación del resguardo hidráulico de un microembalse para repoblación forestal (basado en Ayuso, 1990). 
Con estas justificaciones, debe fijarse un resguardo de varios centímetros sobre la altura de los muretes o un determinado porcentaje de seguridad por encima de la misma, para dotar de mayor seguridad a la estructura en su función de retener toda el agua de escorrentía. Después de la observación de un elevado número de preparaciones del suelo en repoblaciones forestales para la realización de este trabajo, un resguardo de entre el 15 y el $25 \%$ de la altura del murete se estima suficiente para asegurar el cumplimiento de los objetivos marcados. No obstante, la experiencia del técnico es de capital importancia a la hora de fijar esta altura o porcentaje de resguardo.

\section{4) Criterio edáfico de la capacidad de retención de agua disponible}

Como se ha dicho anteriormente, el suelo constituye una reserva de agua cuya medida es la capacidad de retención. Partiendo de una situación inicial en la que esta reserva esté agotada, si durante un aguacero llueve más de lo que el suelo es capaz de retener, el agua sobrante se perderá por percolación profunda hacia la capa freática (este agua recibe la denominación de gravitacional). Por este motivo, el alcorque que se diseñe no debe recoger más agua del que pueda ser almacenado en la reserva del suelo. Por lo tanto, el volumen del alcorque lleno deberá ser, como máximo, igual a la capacidad de retención.

Es difícil, por otra parte, que la reserva llegue a estar totalmente agotada, por lo que casi siempre, inevitablemente, se va a perder agua por percolación profunda. No obstante, en los meses más secos esta reserva será muy escasa mientras no llueva, y por ello, si cae un aguacero convendrá que el alcorque sea capaz de recoger toda el agua necesaria para llenar el depósito edáfico.

Por esta razón deben estar relacionados el volumen de agua que puede contener el alcorque lleno y la capacidad de retención de agua que tiene el suelo abarcado por la rizosfera de las plantas introducidas. Esto equivale a una dosis de riego, si de un cultivo agrícola se estuviera hablando. En conclusión, es necesario que se cumpla la siguiente inecuación:

\section{Volumen alcorque lleno $\leq$ CRAD}

$C R A D=$ Capacidad de retención de agua disponible

El tamaño del alcorque establecido así es suficiente para obtener la máxima cosecha de agua posible (un valor superior quedará fuera del alcance de las raíces al escapar el agua acumulada de la rizosfera)

\section{5) Criterio edáfico de la porosidad}

La altura de los muretes del microembalse y la profundidad del suelo (de la preparación del suelo, de las raíces) son variables que deben estar relacionadas. Si se asocia el criterio edáfico explicado anteriormente con el concepto de porosidad útil, obtenemos la siguiente igualdad (que se explica gráficamente en la figura 3):

\section{Volumen del alcorque lleno $=$ Volumen de poros útiles}

desarrollando:

$$
S_{2} \cdot H=S_{3} \cdot h \cdot p_{\mathrm{u}}
$$

siendo: 


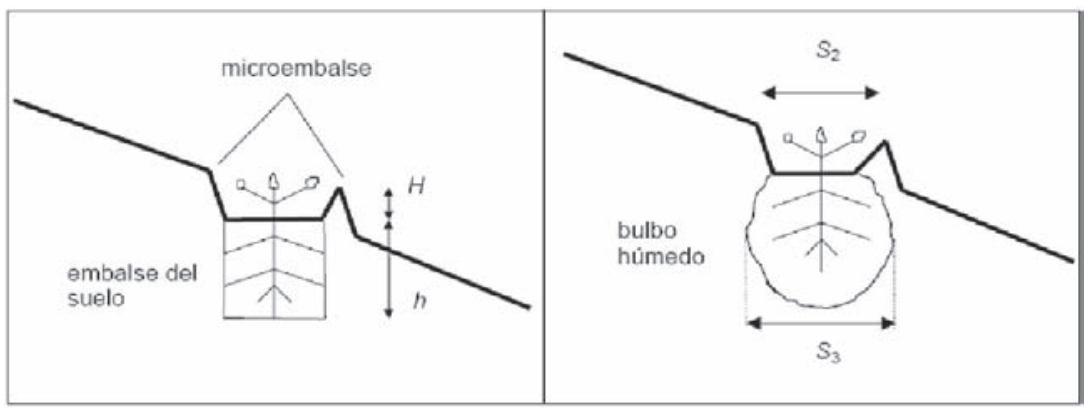

FIGURA 3. Explicación gráfica del criterio edáfico de la porosidad útil.

$S_{2}=$ Área de recepción $\left(\mathrm{m}^{2}\right)$

$H=$ Altura de los muretes (m)

$S_{3}=$ Área que en proyección horizontal ocupa el bulbo húmedo $\left(\mathrm{m}^{2}\right)$, siendo $S_{3} \geq S_{2}$. El «bulbo húmedo» es el nombre que recibe en la terminología del riego localizado el volumen de suelo humedecido por un emisor. Llevado este concepto a las cosechas de agua, la región húmeda del suelo posee una gran importancia, pues en ella se desarrolla el sistema radical de los brinzales. Su forma depende del movimiento del agua en el suelo, que a su vez está condicionado por la textura, la intensidad de precipitación y la duración del aguacero.

$h=\quad$ Profundidad del «embalse del suelo» $(\mathrm{m})$. Para la elección de esta profundidad pueden seguirse tres criterios diferentes: profundidad del sistema radical, profundidad actual del sistema radical más el efecto de la capilaridad y profundidad necesaria para el desarrollo normal del repoblado.

$p_{u}=$ Porosidad útil (en tanto por uno).

Despejando la altura de los muretes:

$$
H=\frac{S_{3}}{S_{2}} \cdot h \cdot p_{U}
$$

Como $S_{3} \geq S_{2}$,

$$
H \geq h \cdot p_{U}
$$

Mediante esta inecuación, es posible calcular la altura de los muretes del microembalse a través de dos variables, que son la porosidad útil del suelo y la profundidad del mismo que se considere deba contener agua utilizable por las plantas.

\section{6) Criterio edáfico de la infiltración}

Siguiendo con la idea de conseguir endorreísmo en la unidad sistematizada, ya enunciada para los criterios hidrometeorológico y de proporcionalidad con el área de impluvio, en este criterio se intenta lograr ese endorreísmo partiendo de la ecuación de infiltración de Horton (1940). En una unidad sistematizada, bajo una precipitación constante, pueden plantearse las siguientes ecuaciones derivadas del modelo de Horton: 
Área de impluvio: $v_{i}(t)=f_{c}+\left(f_{0}-f_{c}\right) \cdot e^{-\alpha \cdot t}$

Área de recepción: $w_{i}(t)=g_{c}+\left(g_{0}-g_{c}\right) \cdot e^{-\beta \cdot t}$

Ante aguaceros prolongados y siempre que $\alpha$ y $\beta$ sean grandes, cabe simplificar estas ecuaciones y admitir:

En el área de impluvio: $v_{i}=f_{c}$

En el área de recepción: $w_{i}=g_{c}$

A su vez y por lo general: $f_{c} \approx g_{c}$ (salvo enmiendas muy importantes en el área de recepción o aplicaciones impermeabilizantes en el área de impluvio, medidas ambas ajenas a las técnicas habituales de reforestación del sector forestal).

La ecuación de continuidad (para sistematizaciones completas), con el fin de conseguir endorreísmo ante una lluvia de intensidad constante y duración $D$, se escribe:

$$
\begin{gathered}
i \cdot D \cdot S \approx f_{c} \cdot D \cdot S_{1}+g_{c} \cdot D \cdot S_{2}+H \cdot S_{2} \\
i \cdot D \cdot S \approx f_{c} \cdot D \cdot\left(S_{1}+S_{2}\right)+H \cdot S_{2} \\
\left(i-f_{c}\right) \cdot D \cdot S \approx H \cdot S_{2}
\end{gathered}
$$

Luego: $H \approx \frac{\left(i-f_{c}\right) \cdot D \cdot S}{S_{2}}=\left(i-f_{c}\right) \cdot D \cdot \kappa$

Acudiendo al modelo clásico de Pérdida Uniforme (por ejemplo, en Aparicio, 1989), en el cual aparece el concepto de tasa uniforme de pérdidas (o índice de infiltración), al que suelen abreviar por $\phi$, podemos escribir:

$$
H \approx(i-\phi) \cdot D \cdot \kappa
$$

siendo, en todo el desarrollo:

$H=$ Altura del microembalse

$v_{i}=$ Velocidad de infiltración de agua en el área de impluvio

$w_{i}=$ Tasa de infiltración de agua en el área de recepción

$f_{0}=$ Capacidad de infiltración inicial en el área de impluvio

$f_{c}=$ Capacidad de infiltración final en el área de impluvio

$\alpha=$ Exponente de decrecimiento para el suelo del impluvio

$g_{0}=$ Capacidad de infiltración inicial en el área de recepción

$g_{c}=$ Capacidad de infiltración final en el área de recepción

$\beta=$ Exponente de disminución para el suelo en el área de recepción

$t=$ Tiempo 
$i=$ Intensidad de lluvia

$D=$ Duración del aguacero

$S=S_{1}+S_{2}=$ Tamaño de la unidad sistematizada

$S_{1}=$ Superficie del área de impluvio $\left(\mathrm{m}^{2}\right)$

$S_{2}=$ Superficie del área de recepción $\left(\mathrm{m}^{2}\right)$

$\kappa=$ Relación entre el tamaño de la unidad sistematizada y el área de recepción $\left(S / S_{2}\right)$

$\phi=$ Tasa uniforme de pérdidas (o índice de infiltración) $\approx f_{c} \approx g_{c}$

\section{7) Criterio ecológico}

En una repoblación forestal, si las plantas arraigan y se desarrollan de forma adecuada, con el paso del tiempo la evolución progresiva de la vegetación lleva emparejada una mejora en las condiciones hídricas y edáficas (Martínez de Azagra, 1996). Estas evoluciones paralelas pueden observarse de forma gráfica en las figuras 4 y 5 . La mejora edáfica se manifiesta por ejemplo en un incremento de la capacidad de infiltración, que implica una disminución del número de curva, lo que puede cuantificarse mediante las tablas de este método (por ejemplo en Ponce, 1989). Se trata, por lo tanto, de conseguir gracias a la preparación del suelo un salto en el número de curva equivalente de la unidad sistematizada $(N E Q)$, que puede llegar incluso a igualar al número de curva clímax $\left(N_{\min }\right)$, es decir, el número de curva de la vegetación climácica de la zona:

$$
N A C>N E Q \geq N_{\text {min }}
$$

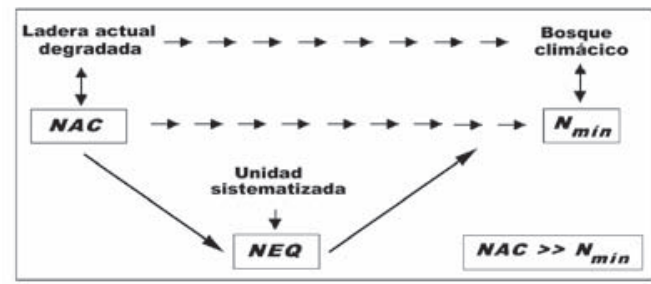

Figura 4. Serie progresiva del número de curva (Martínez de Azagra, 1996).

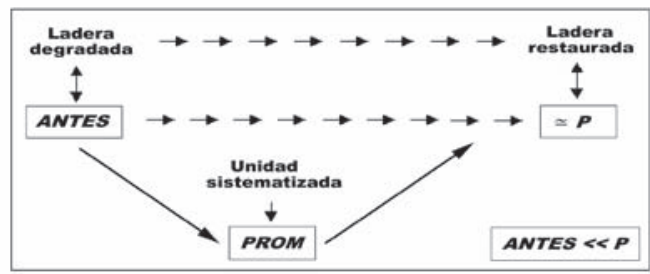

Figura 5. Serie progresiva de las disponibilidades hídricas en una ladera degradada (Martínez de Azagra, 1996). 
Al mismo tiempo, las disponibilidades hídricas en la ladera degradada que inicialmente eran de cuantía muy escasa — variable denominada ANTES en MODIPÉ—, pasan a suponer la totalidad de la precipitación cuando se llega a restaurar totalmente el ecosistema, es decir, cuando se llega al bosque climácico.

El rango de variación del número de curva entre la situación actual degradada $(N A C)$ y la situación final restaurada $\left(N_{\text {min }}\right)$ puede ser muy amplio, partiendo de una ladera gravemente degradada y llegando a la restauración plena de la misma después de un determinado número de años. Según las tablas del número de curva para zonas no urbanas, el mayor cambio posible va de 94 a 15. No obstante, un intervalo más realista para un periodo de tiempo normal (como el mencionado de 50 años), puede ser de 94 a 54, correspondiente respectivamente con un barbecho sobre suelo de tipo D y un bosque en condición hidrológica muy buena sobre suelo de tipo $\mathrm{C}$.

A cada número de curva $N$ le corresponde un umbral de escorrentía $P_{0}$, que se define mediante la ecuación siguiente:

$$
P_{0}=0,2 \cdot \frac{25.400-254 \cdot N}{N}(\mathrm{~mm})
$$

De esta manera se pueden obtener los umbrales de escorrentía tanto para la ladera actual degradada $(P A C)$ como para la ladera restaurada $\left(P_{\text {máx }}\right)$. Estos valores pueden ser relacionados fácilmente con la serie histórica de precipitaciones de la localidad y su correspondiente recurrencia o periodo de retorno (véase la tabla 1). Si el nivel de degradación de la ladera es elevado, el umbral de escorrentía será tan bajo que $t$ representará el número de veces al año en que se produce escorrentía. Por contra, $T$ puede significar un periodo de retorno muy extenso, situación en la cual el suelo de la ladera restaurada funciona como un perfecto sumidero de agua: todo lo que llueve se infiltra o es interceptado, lo que implica una mayor cantidad de agua en el suelo y también la recarga de los acuíferos.

Tabla 1

NÚMERO DE CURVA Y UMBRAL DE ESCORRENTÍA, CON SUS CORRESPONDIENTES PERIODOS DE RETORNO, PARA LA LADERA ACTUAL Y LA RESTAURADA

\begin{tabular}{|c|c|c|c|}
\hline SITUACIÓN & $\begin{array}{c}\text { NÚMERO DE } \\
\text { CURVA }\end{array}$ & UMBRAL DE ESCORRENTÍA & $\begin{array}{c}\text { PERIODO DE } \\
\text { RETORNO }\end{array}$ \\
\hline Ladera actual & $N A C$ & $P A C$ & $t$ \\
\hline Ladera restaurada & $N_{\text {mín }}$ & $P_{\text {máx }}$ & $T$ \\
\hline
\end{tabular}

\section{8) Criterio fisiológico}

El objetivo de este criterio es elegir el tamaño del microembalse de tal forma que sea capaz de almacenar y suministrar un «caudal regulado» equivalente a la evapotranspiración que se produce a lo largo del año. Es decir, se trata de cubrir las necesidades fisiológicas de agua de la repoblación (de ahí el nombre del criterio) con el agua aportada por el alcorque. En este apartado se establece un paralelismo entre un embalse para el abastecimiento de agua de riego o uso humano y un microembalse para repoblación forestal, adaptando para el diseño de este segundo los métodos más habituales de dimensionado del primero (métodos hidráulicos de regulación). 
En el cálculo de la capacidad de un embalse intervienen dos grupos de variables: las aportaciones $(A)$ y las demandas $(D)$. Existen claras diferencias en los componentes de estas variables según se trate de diseñar un embalse o, como es nuestro objetivo, un microembalse para repoblación forestal. En el caso de un embalse habitual, las demandas a considerar serán las necesidades de los regadíos o de consumo humano, mientras que las aportaciones estarán constituidas por el caudal que llega al pantano. En una repoblación forestal, sin embargo, el microembalse deberá compensar con precipitación y recolección de agua las necesidades hídricas de la planta a instalar:

\section{a) Aportaciones}

Como se muestra en la figura 6, que representa el balance hídrico en una ladera con microembalse, las aportaciones que alimentan al alcorque son las precipitaciones sobre el área de recepción $(P)$ y la escorrentía que llega a dicha área $\left(E_{s l}\right)$; recursos que hay que corregir, no obstante, con la intercepción $\left(I_{t}\right)$, la evaporación física desde la superficie del suelo $(E)$ y la escorrentía que escapa de la unidad sistematizada $\left(E_{s 2}\right)$ :

$$
A=P+E_{s 1}-\left(I t+E+E_{s 2}\right)
$$

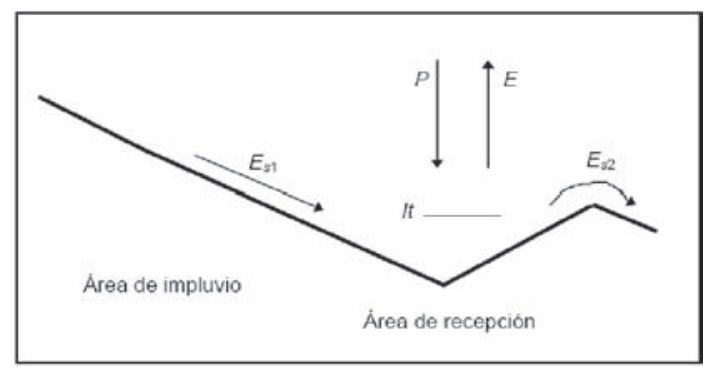

Figura 6. Balance hídrico en una ladera con microembalse.

De todas estas componentes se pueden despreciar la intercepción y la evaporación, por ser de poca cuantía respecto al resto. Tampoco debe ser tenida en cuenta la escorrentía que escapa de la unidad sistematizada, ya que la finalidad del microembalse es retener todo el agua de lluvia y la escorrentía dentro de la unidad. Así pues:

$$
\begin{gathered}
A=P+E_{s 1} \text { en } 1 \cdot \mathrm{m}^{-2} \text { ó bien } \\
A=P \cdot S_{2}+E_{s l} \cdot S_{1} \text { en } \mathrm{l}
\end{gathered}
$$

b) Demandas

El microembalse deberá proporcionar a las plantas un «caudal regulado» igual a las necesidades fisiológicas de agua de la misma. La estimación de estas necesidades es quizás uno de los puntos débiles de los modelos sobre cosechas de agua existentes. En algunos casos, la falta de criterios científicos plenamente contrastados lleva a establecer unos valores empíricos orientativos (Critchley \& Siegert, 1991).

Los requerimientos hídricos de los cultivos agrícolas han sido ampliamente estudiados en los numerosos trabajos publicados sobre diseño agronómico de riegos. En cuanto a especies forestales la situación es bien diferente. Ya sea por las carencias investigadoras 
existentes en el sector, o por la poca importancia que la mayor parte de los científicos forestales han dado a este tema hasta el momento, no se encuentran suficientes estudios al respecto.

No obstante, tanto para cultivos agrícolas como para repoblaciones forestales, los autores coinciden en que la evapotranspiración, es decir, la pérdida de agua en forma de vapor a través de la vegetación y desde la superficie del suelo hacia la atmósfera, resulta fundamental para el conocimiento de las necesidades hídricas de las plantas (Montero de Burgos \& González Rebollar, 1983).

Los vegetales pierden por transpiración, fundamentalmente a través de los estomas de las hojas, la práctica totalidad del agua que absorben por las raíces. El sistema sueloplanta-atmósfera se considera como un continuo hidrodinámico, en el que el agua fluye a favor del gradiente de los potenciales hídricos (de más a menos potencial). Este proceso de absorción y pérdida de agua está controlado fundamentalmente por las raíces y los estomas de las plantas (Sánchez-Toribio, 1992).

La evapotranspiración potencial $(E T P)$ se define como el agua que retorna a la atmósfera en forma de vapor a partir de un suelo completamente cubierto de vegetación y suponiendo que no existe limitación de humedad en el suelo, es decir, en el que exista plena disponibilidad hídrica (Martínez de Azagra \& Navarro, 1996). Es, por lo tanto, un límite superior de la evapotranspiración real $(E T R)$ que realiza una determinada vegetación:

$$
\begin{gathered}
E T R \leq E T P \\
E T R=E T P \cdot k_{c}
\end{gathered}
$$

Siendo $k_{c}$ el denominado coeficiente de cultivo, que será menor o igual a uno. Se trata, por lo tanto, de fijar ese coeficiente que permita transformar la evapotranspiración potencial (hallada por medición directa o por los métodos más usuales: entre otros, Thorthwaite (1948), Blaney-Criddle (1950), Turc (1961), todos ellos citados por Sánchez-Toribio (1992)) en la evapotranspiración real necesaria para la vegetación. En este sentido, existen en la bibliografía valores orientativos de coeficientes de cultivo para diversas especies, pero no existen datos acerca de las especies más interesantes para la restauración de la vegetación en zonas áridas y semiáridas. En la tabla 2 se ofrecen algunos valores de $k_{c}$ correspondientes a especies agrícolas leñosas, con ecología similar a la de las especies que pueden utilizarse en repoblaciones.

Sin embargo, la exigencia de evapotranspiración real que se acaba de establecer resulta excesiva, especialmente en algunas épocas del año, por tratarse de una repoblación forestal y no de un cultivo agrícola a plena actividad vegetativa. En este sentido es útil acudir a la definición de evapotranspiración residual (e), que es la evapotranspiración cuando la savia se para (la actividad vegetativa se detiene) por razón de la sequía (Montero de Burgos \& González Rebollar, 1983). Según estos autores puede considerarse que la relación entre la evapotranspiración potencial y la residual es constante y próxima a 0,2 :

$$
\begin{gathered}
\frac{E T P}{e}=\varphi \approx 0,2 \\
e=E T P \cdot \varphi
\end{gathered}
$$

Para un año completo, puede afirmarse que la evapotranspiración real de la repoblación $\left(E T_{r e p}\right)$ se sitúa entre dos límites:

$$
E T P \cdot k_{c} \geq E T_{r e p} \geq E T P \cdot \varphi
$$


Tabla 2

COEFICIENTES DE CULTIVO (K.) PARA ALGUNAS ESPECIES ECOLÓGICAMENTE PRÓXIMAS A LAS QUE PUEDEN UTILIZARSE EN REPOBLACIONES FORESTALES EN ZONAS ÁRIDAS Y SEMIÁRIDAS

\begin{tabular}{|c|c|c|c|}
\hline \multicolumn{2}{|c|}{ ESPECIE } & $k_{c}$ & FUENTE \\
\hline \multicolumn{2}{|l|}{ Olivo } & 0,55 & Fuentes \& Cruz (1990) \\
\hline \multicolumn{2}{|l|}{ Olivo } & $0,4-0,6$ & Velasco-Molina (1991) \\
\hline \multirow{4}{*}{ Olivo } & Córdoba & $0,45-0,65$ & \multirow{4}{*}{ Barranco et al. (2001) } \\
\hline & Creta & $0,6-0,75$ & \\
\hline & California & $0,55-0,65$ & \\
\hline & California & 0,75 & \\
\hline \multicolumn{2}{|l|}{ Vid } & $\begin{array}{l}0,5 \text { al principio y final del } \\
\text { periodo, } 1 \text { en la mitad }\end{array}$ & Fuentes \& Cruz (1990) \\
\hline \multicolumn{2}{|l|}{ Vid } & $0,55-0,75$ & Velasco-Molina (1991) \\
\hline \multirow{2}{*}{$\begin{array}{l}\text { Almendro y } \\
\text { otros frutales }\end{array}$} & $\begin{array}{l}\text { Con cubierta } \\
\text { vegetal }\end{array}$ & $\begin{array}{l}0,85 \text { al principio y final del } \\
\text { periodo, } 1,15 \text { en la mitad }\end{array}$ & \multirow[t]{2}{*}{ Fuentes \& Cruz (1990) } \\
\hline & $\begin{array}{l}\text { Sin cubierta } \\
\text { vegetal }\end{array}$ & $\begin{array}{c}0,50 \text { al inicio, } 0,90 \text { en la mitad y } \\
0,65 \text { al final }\end{array}$ & \\
\hline
\end{tabular}

Y si se necesita una mayor precisión, es posible establecer un patrón como el que sigue:

- Meses de invierno, siempre que $P \geq E T P: E T_{\text {rep }}=E T P$

- Meses de primavera (2 meses), una vez que $P<E T P: E T_{\text {rep }}=E T P \cdot k_{c}$

- Meses de verano y otoño, hasta que de nuevo $P \geq E T P: E T_{\text {rep }}=E T P \cdot 0,2$

De este modo, los arbolillos crecerán moderadamente durante los dos meses de primavera y sobrevivirán el resto del verano.

Para la aplicación de los métodos que se explicarán posteriormente, es preciso referir las demandas calculadas en $1 \cdot \mathrm{m}^{-2}$ a una determinada superficie para que aparezcan en litros. Esa superficie es la de la proyección de la copa de la planta al final de la vida útil de la sistematización (unos 10 años), que se halla por observación de repoblaciones análogas de la misma especie. Por lo tanto, las demandas se obtendrán mediante el producto de la evapotranspiración real de la repoblación y la mencionada superficie:

$$
D=E T_{r e p} \cdot S_{\text {copalo }}
$$

Una vez conocidas las aportaciones y las demandas, se aplica el método de diagrama de masa. Éste es un método sencillo de dimensionado hidráulico de embalses, desarrollado por 
Rippl (1883) (citado por Zapata, 2001). Consiste en elaborar una tabla de cuatro columnas que contiene la siguiente información:

1) Aportaciones mensuales.

2) Demandas mensuales.

3) Diferencias entre las demandas y las aportaciones.

4) Diferencias positivas acumuladas de la columna anterior.

El mayor valor de esta última columna representa el volumen máximo necesario para satisfacer la demanda y, por lo tanto, será el volumen que deberá tener el microembalse a construir.

\section{9) Limitación fisiológica por encharcamiento}

Después de una precipitación abundante, el alcorque puede llenarse de agua y la planta que en él vegeta quedar anegada, poniéndola en grave riesgo su supervivencia. Con el fin de evitar la muerte por anoxia de la planta, es necesario limitar el tiempo máximo de embalse (Tmáx):

$$
T_{\max } \leq \frac{H}{f_{\min }}
$$

$H=$ Altura de los muretes del microembalse (aproximadamente igual a $C A P A / S_{2}$ ) (cm)

$f_{\text {min }}=$ Tasa de infiltración mínima del suelo en el área de recepción $\left(\mathrm{cm} \cdot \mathrm{h}^{-1}\right)$. Coincide con el parámetro $f$ del modelo de infiltración de Horton (1940). Este parámetro se estima a partir de la permeabilidad, utilizando la relación orientativa propuesta por Bouwer (1966): $f_{\text {min }}=0,5 \cdot k$, siendo $k$ la conductividad hidráulica (o permeabilidad) del suelo.

\section{0) Criterio paisajístico o topográfico}

El movimiento de tierras de la preparación del terreno produce un impacto negativo sobre el paisaje y el suelo. Por este motivo hay que intentar modificar el relieve natural lo mínimo imprescindible, mediante un adecuado diseño de la sistematización primaria, sustentado en la economía del agua.

Así mismo, conviene comprobar que el microrrelieve creado artificialmente se atenúa en pocos años. De esta manera se comprueba que el impacto paisajístico es reversible en poco tiempo. Para ello puede utilizarse una ecuación, derivada de la USLE, que sirve para calcular el volumen de sedimentos que van a parar al microembalse $\left(A_{1}\right)$ :

$$
A_{1}=\frac{R \cdot K \cdot L \cdot S \cdot C \cdot t \cdot S_{1} \cdot 100}{\delta} \cdot\left(1-C_{e}\right)
$$

$R, K, L, S$ y $C=$ Factores de la USLE; $t=$ Vida útil de la sistematización; $S_{1}=$ Área de impluvio; $C_{e}=$ Coeficiente de emisión de sedimentos; y $\delta=$ Densidad aparente de los sedimentos removidos, transportados y depositados en $S_{2}$

Si se cumple la condición siguiente:

$$
C A P A \leq A_{1}
$$


el microembalse resulta completamente aterrado (tras su vida útil) y el relieve artificial generado queda suavizado durante ese tiempo.

Además, la recolección de suelo y nutrientes cuantificada con la ecuación presentada contribuye también a la oasificación de la ladera, puesto que éstos aportan beneficios a las plantas recién instaladas.

\section{1) Criterios mecánicos (estabilidad, asentamiento, estanqueidad)}

Cuando se prepara una sistematización primaria, debe asegurarse siempre la estabilidad y estanqueidad de los muretes de tierra, así como diseñar los muretes teniendo en cuenta el asentamiento de la tierra removida. Unos microrrelieves inestables, además de resultar inútiles como trampas de agua, pueden enterrar a los brinzales provocando su muerte. Así mismo, los muretes no deben dejar pasar agua a su través, cuando el microembalse se encuentre encharcado, ya que esto supondría pérdidas de tan preciado elemento. Tampoco será válida la preparación del terreno si se dimensiona sin considerar que, con el paso del tiempo, verá reducida su altura debido al asentamiento del suelo removido.

\section{$\underline{\text { Estabilidad }}$}

Las preparaciones del suelo pueden ser de dos tipos: aquéllas que se construyen embutidas en el terreno (una poceta o un surco en trinchera, por ejemplo) y las que están constituidas por muretes o caballones elevados sobre el terreno circundante. Desde el punto de vista de la estabilidad son mucho más seguras las primeras, puesto que los taludes excavados en el terreno natural (desmontes) son más estables que los construidos con tierra transportada (terraplén).

En el caso de los muretes o caballones, para que éstos cumplan su función de forma adecuada, debe evitarse en lo posible su desmoronamiento. La estabilidad viene condicionada por las dimensiones del caballón: pendiente de los taludes, anchura en coronación $(e)$, anchura en la base $(b)$ y altura $(H)$ (véase la figura 7).

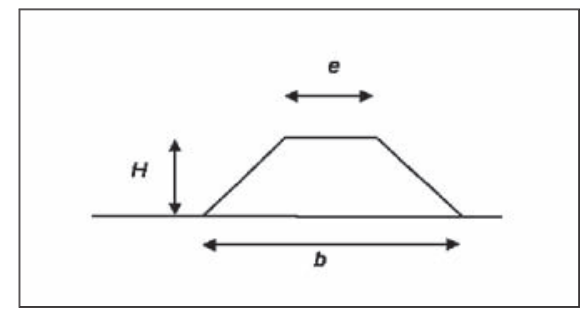

Figura 7. Dimensiones de un murete o caballón.

Respecto a estas dimensiones pueden hacerse algunas recomendaciones:

- Pendiente de los taludes.- La más recomendable es una pendiente 1:1 (Critchley \& Siegert, 1991). Taludes más tendidos no suelen ser necesarios, y sí, en cambio, podrían empinarse más en terrenos arcillosos no conflictivos (hasta llegar por ejemplo hasta $0,5: 1)$.

- Anchura en coronación.- Es aconsejable que se encuentre entre 0,10 y 0,30 m. Critchley \& Siegert (1991) recomiendan 0,25 m.

- Anchura en la base.- Se recomienda que sea: 


$$
b=2 \cdot H+e
$$

- Altura del murete.- No conviene construir caballones menores de 0,25 m, puesto que sería difícil que cumplieran su función. Por otra parte, alturas de murete por encima de $0,75 \mathrm{~m}$ no son recomendables porque suponen un riesgo para su estabilidad.

García Salmerón (1995), aplicando conceptos propios de la mecánica de suelos al movimiento de tierras que se efectúa en los aterrazados, establece diferentes valores para los ángulos que deben tener los taludes de desmonte y terraplén.

Los ángulos de terraplén deben fijarse en base a las características granulométricas del suelo y de su grado de humedad. Si se denomina $i$ al ángulo de inclinación del talud (tg $i$ = altura/base), este ángulo debe ser inferior a un cierto límite llamado ángulo de reposo de tierras, que se define como aquél que toma por sí mismo el material abandonado por tiempo prolongado a la acción de los agentes atmosféricos.

En el diseño de un microembalse y también en su ejecución, conviene determinar el ángulo de inclinación de sus taludes, con el fin de que su funcionamiento hidrológico como elemento óptimo de retención de escorrentía sea compatible con su estabilidad. De esta forma podrá asegurarse que el microembalse cumple su función de forma adecuada durante toda su vida útil. En la tabla 3 se dan los valores orientativos del ángulo de inclinación, en función de la naturaleza del suelo y de su grado de humedad.

El grado de humedad es sumamente importante, ya que si el suelo está completamente seco se pueden admitir mayores inclinaciones, que podrían desmoronarse, sin embargo, en la estación húmeda. Este fenómeno se acentúa en el caso de los suelos arcillosos, porque la presencia mayoritaria de esta fracción granulométrica hace que los suelos se mantengan estables en tiempo seco y que sea más fácil su desmoronamiento cuando están embebidos en agua.

Tabla 3

ÁNGULOS DE LOS TALUDES DE DESMONTE Y TERRAPLÉN, SEGÚN LA NATURALEZA DEL TERRENO, PARA SUELO SECO Y HÚMEDO (MODIFICADA DE GARCÍA SALMERÓN, 1995). SUELO HÚMEDO: COMPLETAMENTE SATURADO DE AGUA, POR ABUNDANTES FILTRACIONES. SUELO SECO: EN CASO CONTRARIO, PERO SE TIENE EN CUENTA LA ACCIÓN NORMAL DE LAS LLUVIAS

\begin{tabular}{|l|c|c|c|c|}
\hline \multirow{2}{*}{ NATURALEZA DEL TERRENO } & \multicolumn{2}{c|}{ TALUD DESMONTE } & \multicolumn{2}{c|}{ TALUD TERRAPLÉN } \\
\cline { 2 - 5 } & $\begin{array}{c}\text { SUELO } \\
\text { SECO }\end{array}$ & $\begin{array}{c}\text { SUELO } \\
\text { HÚMEDO }\end{array}$ & $\begin{array}{c}\text { SUELO } \\
\text { SECO }\end{array}$ & $\begin{array}{c}\text { SUELO } \\
\text { HÚMEDO }\end{array}$ \\
\hline Roca dura & $80^{\circ}$ & $80^{\circ}$ & $45^{\circ}$ & $45^{\circ}$ \\
\hline Roca blanda o fisurada & $55^{\circ}$ & $55^{\circ}$ & $45^{\circ}$ & $45^{\circ}$ \\
\hline Detritus rocosos, guijarros & $45^{\circ}$ & $40^{\circ}$ & $45^{\circ}$ & $40^{\circ}$ \\
\hline Suelo adherente con piedra y tierra vegetal & $45^{\circ}$ & $30^{\circ}$ & $35^{\circ}$ & $30^{\circ}$ \\
\hline Suelo arcilloso, arcillas, margas & $40^{\circ}$ & $20^{\circ}$ & $35^{\circ}$ & $20^{\circ}$ \\
\hline Grava, arena gruesa no arcillosa & $35^{\circ}$ & $30^{\circ}$ & $35^{\circ}$ & $30^{\circ}$ \\
\hline Arena fina, no arcillosa & $30^{\circ}$ & $20^{\circ}$ & $30^{\circ}$ & $20^{\circ}$ \\
\hline
\end{tabular}


Este criterio de estabilidad de los taludes influye directamente en el tamaño del microembalse, ya que si se llega a la conclusión de que la anchura del caballón resulta exagerada, habrá que reducirla, reduciéndose a la vez su altura y, por tanto, el tamaño del alcorque.

\section{$\underline{\text { Asentamiento de la tierra removida }}$}

Cuando se hacen movimientos de tierra en un terreno natural, el volumen aparente de tierra una vez excavada es mayor. Esta variación de volumen se denomina esponjamiento, y se cuantifica por el coeficiente de esponjamiento (García Salmerón, 1995). Este concepto es en realidad la consecuencia de una separación relativa, mayor que la que tenían en su estado natural, de las distintas partículas que constituyen el suelo; es decir, cuanto más se disgregue mayor será su esponjamiento (Dal-Re, 1994). Por el contrario, si los materiales excavados se abandonan a la acción de los agentes atmosféricos, estos disminuyen de volumen y se asientan, es decir, el coeficiente de esponjamiento disminuye. Estos conceptos intuitivos (visualizados en la figura 8) pueden concretarse en forma de ecuaciones:

— Esponjamiento inicial:

— Esponjamiento persistente:

- Asentamiento:

$$
\begin{aligned}
& E=\frac{V-V_{0}}{V_{0}} \\
& E^{\prime}=\frac{V^{\prime}-V_{0}}{V_{0}} \\
& T=\frac{V^{\prime}-V}{V}=\frac{E-E^{\prime}}{1+E^{\prime}}
\end{aligned}
$$
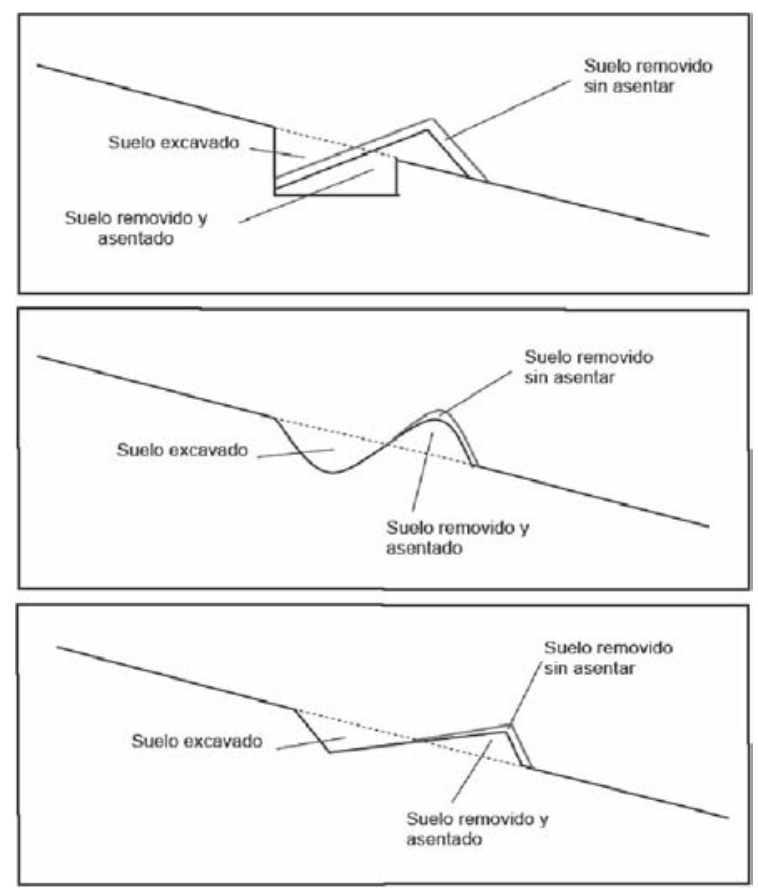

Figura 8. Volúmenes de desmonte y terraplén en diferentes preparaciones del suelo (de arriba abajo: ahoyado, acaballonado o subsolado con rejón modificado, y aterrazado). 
Donde: $V_{0}=$ Volumen de terreno natural.

$V=$ Volumen de los materiales excavados antes de su asentamiento.

$V^{\prime}=$ Volumen después del asentamiento.

Los coeficientes $E, E$ ' y $T$ varían con la naturaleza del terreno, y como orientación pueden utilizarse los aportados por García Salmerón (1995), que se muestran en la tabla 4.

Tabla 4

VALORES DEL COEFICIENTE DE ESPONJAMIENTO (EN TANTO POR UNO) PARA DIFERENTES TIPOS DE TERRENO (MODIFICADO DE GARCÍA SALMERÓN, 1995)

\begin{tabular}{|l|c|c|c|}
\multirow{2}{*}{\begin{tabular}{c}
\multirow{2}{*}{$\begin{array}{c}\text { NATURALEZA } \\
\text { DEL TERRENO }\end{array}$} \\
\cline { 2 - 3 }
\end{tabular}} & \multicolumn{2}{c|}{$\begin{array}{c}\text { COEFICIENTE DE } \\
\text { ESPONJAMIENTO }\end{array}$} & $\begin{array}{c}\text { COEFICIENTE } \\
\text { DE } \\
\text { ASENTAMIENTO } \\
(\boldsymbol{T})\end{array}$ \\
\hline Tierra vegetal, arena & $0,10-0,15$ & $0,01-0,015$ & $0,08-0,12$ \\
\hline Grava & $0,15-0,20$ & $0,015-0,02$ & $0,12-0,15$ \\
\hline Tierra mezclada con arena & $0,20-0,25$ & $0,02-0,04$ & $0,15-0,17$ \\
\hline Tierra arcillosa & $0,25-0,30$ & $0,04-0,06$ & $0,17-0,19$ \\
\hline Arcilla & $0,30-0,35$ & $0,06-0,07$ & $0,19-0,21$ \\
\hline Margas & $0,35-0,40$ & $0,07-0,08$ & $0,21-0,23$ \\
\hline Arcillas y margas muy compactas & $0,40-0,65$ & $0,08-0,15$ & $0,23-0,30$ \\
\hline Conglomerados & $0,30-0,40$ & $0,08-0,15$ & $0,17-0,18$ \\
\hline Roca compacta & $0,40-0,65$ & $0,10-0,25$ & $0,10-0,15$ \\
\hline
\end{tabular}

Los conceptos anteriores pueden aplicarse a preparaciones del terreno en repoblaciones forestales; de hecho, García Salmerón (1995) los aplica a la construcción de terrazas, que requieren grandes volúmenes de desmonte y terraplén. Para construir un murete de tierra o un caballón de volumen definitivo $V$, es necesario obtener un volumen provisional esponjado antes del asentamiento (véase la figura 9), que será:

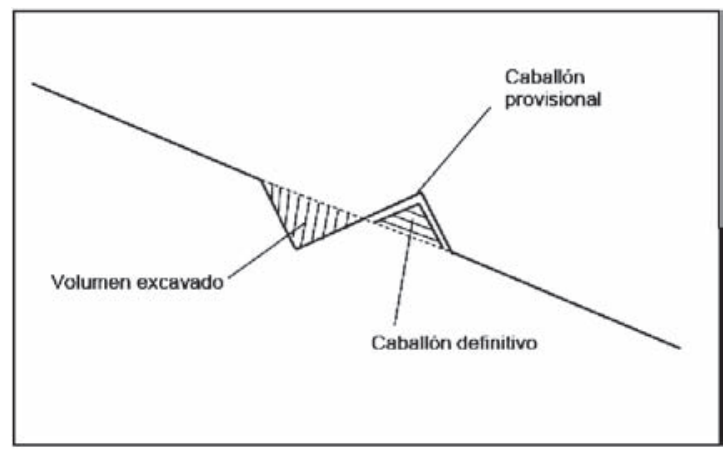

FIGURA 9. Volúmenes definitivo, provisional y de excavación de un caballón. 


$$
V=\frac{V^{\prime}}{1-T}
$$

y, aunque tenga menos interés para el fin que se plantea aquí, deberá excavarse un volumen natural:

$$
V_{0}=\frac{V}{1+E}
$$

$\underline{\text { Estanqueidad }}$

$\mathrm{Al}$ igual que las grandes presas de tierra presentan pérdidas por filtración, las micropresas que constituyen las estructuras de recogida de agua en repoblaciones forestales pierden agua, al estar conformadas por un material poroso y, casi siempre, sin compactar. Estás pérdidas de agua en suelo saturado, cuando el microembalse está encharcado, pueden cuantificarse mediante la ley de Darcy (1865). Lógicamente, las pérdidas de agua fuera del microembalse, por pequeño volumen que supongan, no son deseables para el objetivo de dotar al árbol de una buena cantidad de este elemento. Sin embargo, debido al ámbito en el que se trabaja, no es necesario el cálculo con precisión de estas salidas, y bastará con tomar algunas sencillas medidas, basadas en las apreciaciones que se hacen a continuación.

En las técnicas de riego por gravedad, el agricultor construye unos caballones de tierra entre surcos por los que circula el agua, de una anchura tal que no traspase el agua al surco contiguo, para conseguir que la mayor cantidad de agua posible se infiltre en el surco, dentro del cual está establecido el cultivo.

De la misma forma, el caballón o murete de una preparación del terreno para repoblación debe ser lo suficientemente ancho como para no tener pérdidas de agua por flujos laterales. En otras palabras, el caballón debe ser estanco para que toda el agua que llega al área de recepción se infiltre en la misma. En este proceso tiene influencia tanto el suelo removido del caballón (su capacidad para transmitir agua o conductividad hidráulica, como se vio anteriormente) como el suelo sin alterar que se encuentra justo debajo del área de recepción (que determinará la infiltración).

En suelos arenosos el agua penetra más en profundidad que lateralmente. Por el contrario, en arcillosos el agua penetra con más rapidez en sentido horizontal que en vertical. Por este motivo, en riegos por gravedad se recomienda que la separación entre surcos (o lo que es lo mismo, la anchura del caballón) no sea mayor de $50 \mathrm{~cm}$ en suelos arenosos, $100 \mathrm{~cm}$ en suelos de textura media y $150 \mathrm{~cm}$ en suelos arcillosos (Fuentes, 1998). El suelo del caballón ha sufrido cambios en su estructura (está removido), hecho que favorece la conductividad hidráulica (Porta et al., 1999). Por otra parte, cuanto mayor sea el tiempo de encharcamiento del microembalse mayor posibilidad existe de que el agua escape de forma lateral.

A nivel práctico, cuanto más arenoso sea un suelo, más estrecho puede ser el caballón, y cuanto más arcilloso más ancho debe ser. Si con este criterio la anchura del caballón resulta inconveniente, habrá que reducir la altura del mismo, por consiguiente, también disminuirá el tamaño del microembalse.

\section{2) Criterio económico}

La preparación del terreno, el refinado y la conformación del alcorque no deben incrementar de manera considerable los costes de la repoblación. No obstante, el sobrecoste que supone diseñar un buen sistema de recolección de agua se justifica en los siguientes casos: 
— Intentos infructuosos de repoblación por métodos tradicionales

- Peligro para poblaciones o asentamientos humanos

- Riesgo para infraestructuras (presas, carreteras, vías de ferrocarril, puentes, etc.), grandes pérdidas económicas

- Zona muy degradada que requiera de todos los esfuerzos para su restauración (concepto de área crítica)

\section{Integración de los criterios de diseño}

Una vez analizados los criterios propuestos para el diseño de microembalses de manera individualizada, es conveniente dar algunas indicaciones acerca del orden más adecuado de efectuar los cálculos y de la forma correcta de integrar todos los criterios (véase la figura 10). Para ello, se han agrupado estos criterios de la forma siguiente:

a) Criterios fundamentales.- Se trata de criterios que sirven como punto de partida al método propuesto de dimensionado del microembalse, y comprenden condiciones o principios de vital importancia en el diseño. Criterios hidrometeorológico, de resguardo hidráulico, proporcionalidad con el área de impluvio, ecológico.

b) Criterios de referencia.- Son también importantes, por lo que se consideran como referentes en el diseño del microembalse. Edáficos, paisajístico, limitación fisiológica, fisiológico.

c) Criterios recomendatorios.- Simplemente aconsejan modificaciones del tamaño del embalse en función de aspectos relevantes de diseño, pero no fundamentales. Mecánicos.

d) Criterio económico.- Este criterio, al no ser meramente técnico, se sitúa al final del proceso, condicionando la decisión definitiva.

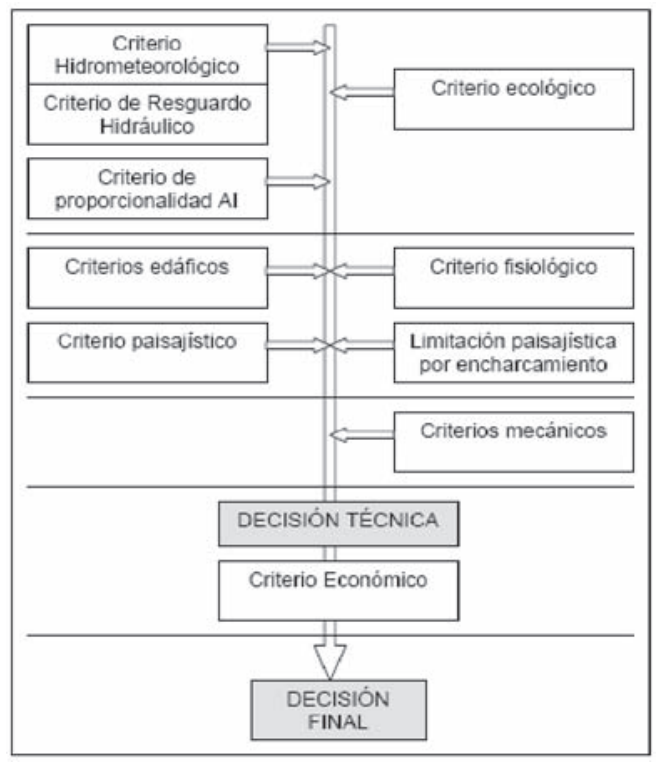

FiguRa 10. Integración de los criterios de diseño del microembalse. 


\section{Relación entre área de impluvio y área de recepción}

En las repoblaciones forestales la relación entre el área de impluvio y el área de recepción viene determinada, habitualmente, por la decisión sobre el método de preparación del suelo y la densidad de plantación. Sin embargo, para zonas áridas y semiáridas cabe definir criterios al respecto, basados en la economía del agua, que tendrán su influencia sobre la densidad de la repoblación finalmente lograda. Se trata, por tanto, de un proceso inverso de cálculo, donde la densidad no es un dato de partida y sí la disponibilidad hídrica en el área de recepción.

La relación $S_{1} / S_{2}$ óptima puede obtenerse de forma directa por experimentación en campo para unos determinados cultivos (o, en nuestro caso, para unas determinadas especies forestales), o indirectamente mediante la utilización de modelos matemáticos desarrollados con este fin. Existen numerosos trabajos que aportan información orientativa al respecto. Así, Boers \& Ben-Asher (1982) encuentran tamaños de microcuencas comprendidos entre 0,5 y $1.000 \mathrm{~m}^{2}$, para árboles, arbustos y cultivos. Aseguran estos autores que la relación entre el área de contribución y el área de infiltración, varía entre 1 y 6, dependiendo del clima, de las características edáficas y de los requerimientos hídricos del cultivo. Por su parte, Critchley \& Siegert (1991) recogen datos de microcuencas para uso arbóreo, de zonas tan distantes como Kenia, Israel o India, que van desde cocientes entre 0,9 (para Ziziphus mauritiana en India) y 64 (para especies productoras de leñas en Nigeria). Recomendaciones similares hacen Tadmor et al (1960), para Israel, Finkel (1985), en Kenia, o Prinz (1999) en India. En México se han realizado también diversas experiencias, destacando las de Martínez \& Cepeda (1970), Gutiérrez (1975) y Carranza (1978).

Por otra parte, se han revisado algunos de los modelos existentes a nivel mundial, de carácter agrícola fundamentalmente, para definir la relación óptima o necesaria entre el área de impluvio y el área de recepción. Los métodos analizados han sido: Anaya et al. (1976), Smith (1978), Velasco-Molina (1983), Finkel (1985), Critchley \& Siegert (1991), Velsavill (Villanueva et al., 1987), Cadot (1989), Palomo (1990) y Oweis et al. (1999). Sus principales características se pueden resumir en los siguientes puntos:

- Se basan en la compensación de las necesidades del cultivo por la precipitación que cae en el área de recepción y la escorrentía generada en el área de impluvio.

- Tienen carácter agrícola (a excepción del método para árboles desarrollado por Critchley \& Siegert (1991).

- Son de aplicación sencilla

- Estiman las necesidades hídricas a través de la ETP

- Calculan la escorrentía mediante coeficientes de escurrimiento

- A menudo emplean balances hídricos demasiado precisos para lo que se requiere en el diseño de repoblaciones forestales.

El método de Finkel (1985) es posiblemente el más empleado a nivel mundial, y se resume en la siguiente ecuación:

$$
\frac{C}{C A}=\frac{R H-P D}{P D \cdot k \cdot E}
$$

donde: $\quad C=$ Área de captación (o de impluvio)

$C A=$ Área de cultivo (o de recepción)

$R H=$ Requerimientos de agua que tiene el cultivo

$P D=$ Precipitación de diseño 
$k=$ Coeficiente de escorrentía

$E=$ Factor de eficiencia

Fundamentado en la economía del agua, la idea principal del método que se presenta es que el tamaño del área de impluvio debe ser el adecuado para proporcionar una determinada cantidad de agua para permitir (o asegurar) la subsistencia del repoblado durante su fase más crítica (arraigo y primer crecimiento). Es decir, se pretende que exista una dotación de agua suficiente en el área de recepción gracias al área de impluvio; bajo la premisa de que se produce un mayor aporte de agua a mayor área de impluvio (véase la figura 11).

El método que se propone parte del siguiente balance hídrico:

$$
E_{S 1} \cdot S_{1}+P \cdot S_{2}=E T_{\text {rep }} \cdot S_{2}
$$

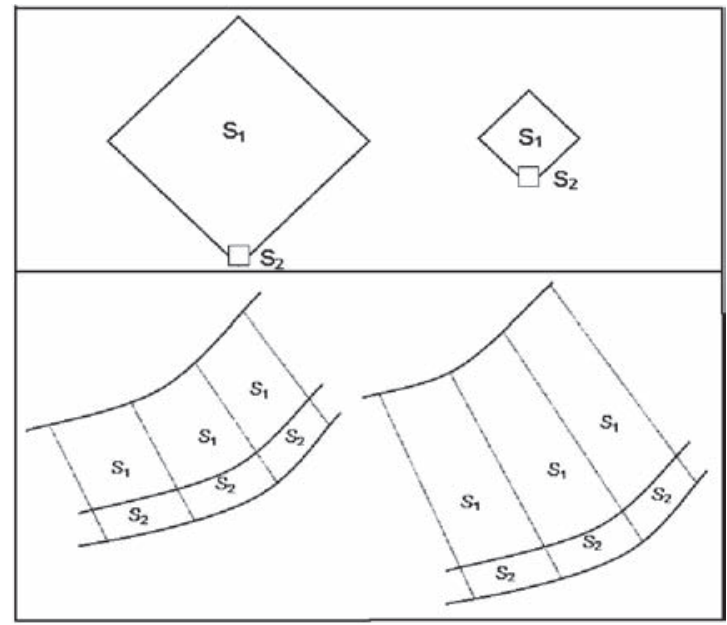

Figura 11. En las preparaciones del suelo, como las microcuencas (arriba) u operaciones lineales (abajo), el área de impluvio $\left(S_{1}\right)$ debe tener un tamaño adecuado para dotar de agua suficiente al área de recepción $\left(S_{2}\right)$.

Siendo:

$E_{S 1}=$ Escorrentía formada en el área de impluvio que llega al área de recepción $\left(1 \cdot \mathrm{m}^{-2}\right)$

$P=$ Precipitación de diseño $\left(1 \cdot \mathrm{m}^{-2}\right)$

$E T_{\text {rep }}=$ Evapotranspiración real de la repoblación $\left(1 \cdot \mathrm{m}^{-2}\right)$

$S_{1} \stackrel{\text { rep }}{=}$ Área de impluvio $\left(\mathrm{m}^{2}\right)$

$S_{2}=$ Área de recepción $\left(\mathrm{m}^{2}\right)$

Si del balance anterior se despeja la relación entre el área de impluvio y el área de recepción $\left(R=S_{1} / S_{2}\right)$, queda la siguiente ecuación:

$$
R=\frac{S_{1}}{S_{2}}=\frac{E T_{r e p}-P}{E_{S 1}}
$$

Para no dar lugar a equívocos, conviene explicar cada uno de los componentes de la ecuación: 
a) Evapotranspiración real de la repoblación.- La evapotranspiración de la repoblación se considera en este método estimadora de los requerimientos hídricos de las plantas, al igual que hacen otros autores como Smith (1978), Velasco-Molina (1983), Cadot (1989), Palomo (1990) u Oweis et al. (1999), o con otro tipo de fines Thornthwaite \& Matter (véase, por ejemplo, en Martínez de Azagra \& Navarro, 1996) en su balance hídrico y Montero de Burgos \& González Rebollar (1983) en los diagramas bioclimáticos, entre otros.

La estimación de la evapotranspiración real de las especies forestales (y especialmente de una repoblación) es, en la actualidad, poco precisa; por ello será necesario profundizar en el tema en futuros trabajos de investigación.

¿Cuál es entonces la medida de los requerimientos de agua de la repoblación? La respuesta a esta pregunta hay que buscarla en la propia Naturaleza. Aquellos años en los que se produzca una exitosa regeneración en las masas naturales análogas a la que se quiere crear, tendrán unas determinadas precipitaciones y una gran cantidad de semillas germinadas, y se habrá producido una evapotranspiración real que será idónea para satisfacer las necesidades de las nuevas plantas instaladas. Sin embargo, esta forma de proceder es bastante poco operativa a nivel general, por lo que una aproximación práctica y del lado de la seguridad, que llevará al dimensionado de áreas de impluvio de superficie holgada para cubrir las demandas del repoblado, puede ser la ETP. Por otra parte, es la variable que utilizan la mayor parte de los métodos que en la revisión bibliográfica se han descrito.

b) Precipitación de diseño.- Se utiliza como tal la precipitación anual que tiene una probabilidad de ocurrencia del $33 \%$ (equivalente a un periodo de retorno de 1,5 años, lo que supone que, de media, dos de cada tres años lloverá más que la precipitación calculada), determinada mediante el ajuste de los datos de precipitación anual de la serie disponible a la función de distribución de Goodrich (recomendada para valores medios).

c) Escorrentía en el área de impluvio.- La escorrentía que se forma en el área de impluvio y que contribuye a incrementar las disponibilidades hídricas del área de recepción interesa estimarla por el método del número de curva, puesto que es más preciso y recoge una mayor cantidad de situaciones que las tablas habituales de coeficientes de escorrentía.

De acuerdo con el método de los complejos hidrológicos, la escorrentía se calcula como:

$$
E_{S 1}=\frac{(P-P 1)^{2}}{P+4 \cdot P 1}
$$

Siendo:

$P 1=$ Umbral de escorrentía en el área de impluvio $(\mathrm{mm}): P 1=0,2 \cdot \frac{25.400-254 \cdot N I}{N I}$
$N I=$ Número de curva del área de impluvio

$P=$ Precipitación de un aguacero individual $(\mathrm{mm})$. En este caso hay que considerar todos los aguaceros que ocurren en el año de cálculo (serie real de aguaceros) o, en su defecto, utilizar una distribución de precipitaciones obtenida por el método de los aguaceros virtuales (a este respecto puede consultarse Martínez de Azagra, 1996). Existen tres casos posibles de distribución de las precipitaciones, que generan respectivamente una escorrentía máxima, mínima y media.

En aras a permanecer del lado de la seguridad en la estimación de la relación entre el área de impluvio y el área de recepción, puede hacerse una modificación a la ecuación expresada anteriormente para el cálculo de $R$. Esta variación consiste en sustituir la precipitación de diseño por la precipitación efectiva, como hacen Villanueva et al. (1987) y Palomo (1990), e incluir un factor de eficiencia, empleado por Finkel (1985) y Critchley \& Siegert (1991): 
- Precipitación efectiva $\left(P_{e}\right)$.- La precipitación efectiva es la fracción de la precipitación que puede llegar a estar disponible en la zona del suelo ocupada por las raíces de las plantas. Las factores que la determinan son: intensidad, duración y distribución de la lluvia, velocidad de infiltración del suelo, cubierta vegetal y topografía (Velasco-Molina, 1991). Para el cálculo de la precipitación efectiva se parte de la precipitación de diseño (con probabilidad de ocurrencia del $33 \%$ ) y se utiliza alguno de los métodos aplicables a regiones secas, por ejemplo el de Blaney-Criddle, que aconseja Velasco-Molina (1991).

- Factor de eficiencia $(E)$.- Este coeficiente cuantifica la desigual distribución del agua de escorrentía sobre el terreno, así como las pérdidas debidas a la evaporación y a la percolación profunda. La elección del factor de eficiencia es una decisión que debe tomar el técnico que proyecta la repoblación en base a su experiencia y a la técnica de sistematización elegida. Finkel (1985) considera factores de eficiencia entre 0,25 y 0,85; mientras que Critchley \& Siegert (1991) utilizan un valor de 0,5 para métodos de preparación del suelo con áreas de impluvio grandes (por ejemplo, caballones trapezoidales) y de 0,75 para técnicas que implican pequeñas áreas de impluvio (como es el caso de microcuencas Negarim o caballones según curvas de nivel).

Estos dos parámetros, precipitación efectiva y factor de eficiencia, aumentan $R$ (y en consecuencia, disminuyen la densidad de plantación). Su empleo puede parecer arbitrario e innecesario, e incluso puede producir cierta incertidumbre en el cálculo de $R$. No obstante, su utilidad radica en que introduce una ecuación que calcula una $R$ mayor, que crea un intervalo de confianza a la hora de elegir la $R$ definitiva de la repoblación. Así pues, se puede calcular la relación entre el área de impluvio y el área de recepción por la ecuación vista anteriormente:

$$
R_{\text {min }}=\frac{E T_{r e p}-P}{E_{S 1}}
$$

Y también, empleando la precipitación efectiva y el factor de eficiencia:

$$
R_{\text {mayor }}=\frac{E T_{r e p}-P_{e}}{E_{S 1} \cdot E}
$$

Esta segunda ecuación permite situarse del lado de la seguridad respecto a la cantidad de agua de escorrentía que se aporta a las plantas, y por tanto garantiza con mayor probabilidad de éxito la supervivencia de la repoblación.

\section{Bibliografía}

ANAYA, G.M.; TOVAR, S.; TOVAR, A.; MACÍAS, L. (1976). Métodos de captación de lluvia para zonas agrícolas con temporal deficiente. Colegio de Postgraduados de Chapingo. Chapingo.

AYUSO, J.L. (1990). Circulación de flujos. Métodos de cálculo usuales en el diseño de canales y embalses en cuencas pequeñas. Monografía $\mathrm{n}^{\circ} 179$. Servicio de publicaciones de la Universidad de Córdoba. Córdoba.

BARRANCO, D.; FERNÁNDEZ-ESCOBAR, R.; RALLO, L. (Eds.) (2001). El cultivo del olivo. Ed. Mundi-Prensa. Madrid.

BOERS, TH. M.; BEN-ASHER, J. (1982). «A review of rainwater harvesting». Agric. Water Manage., 5: 145-158.

BOUWER, H. (1966). «Rapid field measurement of air-entry value and hydraulic conductivity of soil as significant parameters in flow system analysis». Water resources research, 2: 729-738.

CADOT, P.D. (1989). Development of a model for design of water harvesting systems in small scale rainfed agriculture. University of Arizona. U.M.I. Ann Arbor. 
CARRANZA, P.A. (1978). Establecimiento de huertos frutales con aguas de escurrimiento en pequeñas cuencas hidrológicas. Avances de Investigación UAAN. Saltillo.

CRITCHLEY, W.; SIEGERT, K. (1991). Water harvesting. Food and Agriculture Organization of the United Nations. Roma.

DAL-RE, R. (1994). Caminos rurales. Proyectos y construcción. MAPA-IRYDA-Mundi-Prensa. Madrid.

FINKEL, M. (Ed.) (1985). Turkana water harvesting manual. Finkel and Finkel Ltd. Norad.

FUENTES, J.L. (1998). Técnicas de riego. Ed. Mundi-Prensa. Madrid.

FUENTES, J.L.; CRUZ, J. (1990). Curso elemental de riego. Madrid.

GARCÍA SALMERÓN, J. (1995). Manual de repoblaciones forestales II. E.T.S. de Ingenieros de Montes (Fundación Conde del Valle de Salazar). Madrid.

GUTIÉRREZ, C.J. (1975). Evaluación de diferentes métodos para establecimiento de especies forestales en zonas áridas. Tesis. Universidad Autónoma Agraria «Antonio Narro». México D.F.

HORTON, R.E. (1940). «An approach toward a physical interpretation of infiltration capacity». Soil Science Society of America Proceedings, 5: 399-417.

MARTÍNEZ DE AZAGRA, A. (1995). Modelo para la estimación de las disponibilidades hídricas en ladera. Tesis doctoral. E.T.S.I. Montes. Madrid.

MARTÍNEZ DE AZAGRA, A. (1996). Diseño de sistemas de recolección de agua para la repoblación forestal. Ed. Mundi-Prensa. Madrid.

MARTÍNEZ DE AZAGRA, A. (2000). «Principles for designing endorheic microcatchments». Third International Congress Man and Soil at the Third Millennium, Volumen I: 507-520.

MARTÍNEZ DE AZAGRA, A.; CALVO, M. (1996). «Desertización por aridez edáfica». Actas del I Congreso Regional del Agua (Valladolid): 111-117.

MARTÍNEZ DE AZAGRA, A.; MONGIL, J. (2001). «Algunos criterios para el diseño de sistemas de recolección de agua en repoblaciones forestales». Actas III Congreso Forestal Español, Mesa 3: $272-277$

MARTÍNEZ DE AZAGRA, A.; MONGIL, J. ROJO, L. (2004). «Oasification: reclamation forestry addressing desertification hazards». En: NATO-CCMS and Science Committee Workshop on Desertification in the Mediterranean Region. A security issue. Kluwer Academic Publishers.

MARTÍNEZ DE AZAGRA, A.; NAVARRO, J. (1996). Hidrología forestal. El ciclo hidrológico. Servicio de publicaciones de la Universidad de Valladolid. Valladolid.

MARTÍNEZ, M.L.; CEPEDA, V.S. (1970). «Aprovechamiento de agua de escurrimiento y producción de cosechas en suelos de laderas aluviales de la zona árida del municipio de Saltillo». Boletín Técnico ESAAN. Saltillo.

MONTERO DE BURGOS, J.L.; GONZÁLEZ REBOLLAR, J.L. (1983). Diagramas bioclmáticos. Ministerio de Agricultura, Pesca y Alimentación (Instituto para la Conservación de la Naturaleza). Madrid.

OWEIS, TH.; HACHUM, A.; KIJNE, J. (1999). Water harvesting and supplemental irrigation for improved water use efficiency in dry areas. SWIM Papers. Colombo.

PALOMO, M. (1990). Obtención del intervalo de siembra entre bordos antierosivos mediante un modelo simplificado de balance hídrico para zonas semiáridas. Tesis de Maestría. Colegio de Graduados- Escuela Superior de Agricultura «Hermanos Escobar». Ciudad Juárez.

PONCE, V.M. (1989). Engineering hydrology. Principes and practices. Ed. Prentice Hall. Englewood Cliffs.

PORTA, J.; LÓPEZ-ACEVEDO, M.; ROQUERO, C. (1999). Edafología. Para la agricultura y el medio ambiente. Ed. Mundi-Prensa. Madrid.

PRINZ, D. (1999). Technological potential for improvements of water harvesting. WCD Thematic Review IV.2 Assessment of Irrigation Options.

SMITH, G.L. (1978). Water harvesting technology applicable to semiarid, subtropical climates. Agency for International Development. Washington D.C.

TADMOR, N.H.; SHANAN, L.; EVENARI, M. (1960). «The ancient desert agriculture of the Negev. IV. The ratio of catchment to cultivated area». Ktavim, 10: 3-4.

VELASCO-MOLINA, H.A. (1983). Uso y manejo del suelo. Ed. Limusa. México D.F. 
VELASCO-MOLINA, H.A. (1991). Las zonas áridas y semiáridas. Sus características y manejo. Ed. Limusa. México D.F.

VILLANUEVA, J.; SÁNCHEZ, I.; VELASCO-MOLINA, H. (1987). «A method to determinate the relation of water harvesting area to planting area on seasonal crops». Symposium on strategies for classification and management of native vegetation for food production in arid zones, Tucson (Arizona).

ZAPATA, A.J. (2001). Hidrología. Servicio de Publicaciones de la Universidad de Almería. Almería. 